\title{
Short hairpin RNA targeting survivin inhibits growth and angiogenesis of glioma $\mathrm{U} 251$ cells
}

\author{
HAI-NING ZHEN ${ }^{1 *}$, LI-WEN LI ${ }^{2 *}$, WEI ZHANG ${ }^{1}$, ZHOU FEI $^{1}$, CHANG-HONG SHI ${ }^{1}$, \\ TONG-TAO YANG ${ }^{1}$, WEN-TAO BAI ${ }^{1}$ and XIANG ZHANG ${ }^{1}$ \\ ${ }^{1}$ Institute of Neurosurgery, Xijing Hospital, Fourth Military Medical University, Xi'an 710033; \\ ${ }^{2}$ Department of Bioscience, College of Life Sciences, Northwest University, Xi'an 710069, P.R. China
}

Received May 18, 2007; Accepted July 23, 2007

\begin{abstract}
Survivin is a novel tumor-associated gene, its overexpression mostly associates with carcinogenesis and development. Nevertheless, the precise role of survivin in initiation and progression of gliomas is still not completely clear. We constructed here three short hairpin RNA (shRNA) targeting survivin plasmid vectors and introduced them into glioma U251 cells. The three shRNAs were efficiently and specifically able to knockdown the survivin expression in transiently transfected U251 cells. The stable transfectants expressing the shRNA having the strongest inhibitory effect against survivin exhibited decreased cell growth, increased spontaneous apoptosis, mitotic catastrophe and cell cycle arrest. Furthermore, in nude mice xenografts, the stable transfectants presented decreased de novo glioma formation and reduced development of angiogenesis. Results from this study indicate that survivin plays an important role in malignant proliferation, antiapoptosis and angiogenesis of gliomas, which may become an attractive target for gene therapy of gliomas, while RNA interference (RNAi) mediated by shRNA may become a new promising strategy for cancer gene therapy.
\end{abstract}

\section{Introduction}

Deregulation of cell proliferation and cell apoptosis and angiogenesis underlie neoplastic initiation and development, which involves multiple gene alterations, regulated by complicated signal transduction pathways $(1,2)$. The inhibitor

Correspondence to: Dr Xiang Zhang, Institute of Neurosurgery, Xijing Hospital, Fourth Military Medical University, Xi'an 710033, P.R. China

E-mail: zhenhn@fmmu.edu.cn

${ }^{*}$ Contributed equally

Key words: survivin, short hairpin RNA, glioma, growth, angiogenesis of apoptosis protein (IAP) family is an important factor in inhibiting apoptosis, characterized by the presence of one or more 70-80 amino acid baculoviral IAP repeat (BIR) domains at the amino terminal, possessing or not a carboxyl terminal RING zinc-finger. IAPs can directly bind caspases and inhibit their activities in inducing apoptosis, and thus play a critical role in deciding cell fate. Up to now, eight mammalian IAPs have been discovered, i.e. neuronal apoptosis-inhibitory protein (NIAP), cellular IAP-1 (cIAP-1), cIAP-2, X-linked IAP (XIAP), IAP-like protein-2 (ILP-2), Apollon, livin and survivin (3).

Survivn is a novel member of IAP family, first identified by Ambrosini et al (4). The survivin gene is located on human chromosome 17q25.3, encoding a 16.5-kDa protein comprising 142 amino acids. Survivin obviously differs from the other IAP members in several aspects (5-7). Structurally, survivin is the smallest member of mammalian IAP family, containing only one BIR domain, and lacking the RING structure, which substituted by a long amphipathic $\alpha$ helix, thus leading survivin to generate a 'bow-tie' shape in the dimer (8). Functionally, besides the common antiapoptotic activity held by IAPs (9-11), survivin also plays an important role in maintaining normal mitosis $(12,13)$, promoting cell proliferation (14) and facilitating angiogenesis (15-17). Survivin is generally expressed in embryonic tissues, but is either undetectable or expressed at a very low level in terminally differentiated adult tissues, whereas it is overexpressed in the majority of human cancers. Moreover, overexpression of survivin in cancers mostly correlates with enhanced tumor malignant degree, shorter patient survival, accelerated rate of recurrence, and increased resistance to therapy (5-7). All these unique features of survivin make it a potential target with respect to molecular therapy of cancers (5-7). In fact, it shows that functional interference with survivin using dominant-negative mutants, such as survivin Thr34Ala (18) and survivin Cys84Ala (19), as well as attenuation of survivin expression using antisense oligonucleotides $(20,21)$, antisense RNAs (19), ribozymes $(22,23)$ or RNAi strategy $(24,25)$ can indeed induce apoptosis and inhibit growth in cancer cells, and sensitize cancer cells to anticancer modalities, such as chemotherapy and radiotherapy. However, most of the studies regarding the role of survivin in apoptosis and cell division are performed using the transient expression 
method, and the in vivo mechanism of targeting survivin in cancer treatment is not fully understood. Especially, the role of survivin in tumor angiogenesis has not been fully elucidated.

Gliomas are among the most aggressive of all human malignancies. Patients with the most malignant histopathologic subtype, glioblastoma (multiforme), carry the worst prognosis, with median survival less than one year, despite aggressive surgery with adjuvant radiotherapy and chemotherapy (26). It has been shown that survivin is overexpressed in gliomas, and overexpression of survivin is associated with tumorigenesis and progression of gliomas, as well as the poor prognosis of patients with gliomas (27-30). Moreover, our previous study shows that survivin closely correlates to excess proliferation, antiapoptosis and angiogenesis in gliomas (31). Nevertheless, the real role of survivin in malignant biologic behaviour of gliomas is still not completely clear.

In the present study, we constructed specific shRNA targeting survivin plasmid vectors using RNAi technique, introduced them into human glioma U251 cells, established stable transfectants expressing the shRNA against survivin, and further investigated the effects of survivin downregulation mediated by shRNA on the growth, proliferation, apoptosis and angiogenesis of glioma U251 cells in vitro and in nude mice xenografts.

\section{Materials and methods}

Oligonucleotides and plasmids. Using Vector NTI Suite 10.0 package (InforMax, Frederick, MD, USA), according to the survivin cDNA coding sequence (GenBank accession no. NM 001168.2) and the requirements for vector design, three pairs of 64-nuleotide long oligonucleotides were designed and synthesized (Sangon Biotechnology, Shanghai, China), which respectively contain a 19-nucleotide long specific survivin sequence, i.e. SR1: 5'-GAG CCA AGA ACA AAA TTG C-3' (positions 444-462), SR2: 5'-GAA AGT GCG CCG TGC CAT C-3' (positions 508-526), and SR3: 5'-GAA CTG GCC CTT CTT GGA G-3' (positions 190-208). The SR2 sequence was scrambled to form a non-specific shRNA as a control. The oligonucleotides were annealed, digested and cloned into BglII and HindIII sites of pWH1 plasmid vector (Sangon), ensuring the inserted sequence was immediately downstream of the U6 promoter, to construct the specific shRNA vectors and non-specific shRNA vector, which were named pWH1-SR1, pWH1-SR2, pWH1-SR3, and pWH1-NR respectively. The precision of the constructs was confirmed by restriction enzyme digesting and sequencing.

Cell culture and transfection. U251 cells were purchased from American Type Culture Collection (Manassas, VA, USA) and were maintained under conditions recommended by this organization. Transient and permanent transfection experiments were performed using Lipofectamine 2000 (Invitrogen Life Technologies, Grand Island, NY, USA) according to the manufacturer's instructions. In transient transfections, cells were harvested $72 \mathrm{~h}$ after transfection, subjected to reverse transcription-polymerase chain reaction (RT-PCR) and Western blot experiments to determine the
shRNA vector having the strongest efficiency in inhibiting survivin expression. In permanent transfections, after transfection of the shRNA vector having the strongest inhibitory effect and the non-specific pWH1-NR vector, U251 cells were screened with Geneticin (Invitrogen) at $800 \mu \mathrm{g} / \mathrm{ml}$ for 4 weeks. The stable transfectants were named U251-SR and U251-NR respectively, and maintained in medium containing $400 \mu \mathrm{g} / \mathrm{ml}$ Geneticin for additional studies.

RT-PCR and Western blotting. Total RNA of cells was extracted with RNeasy Mini Kit (Qiagen, Valencia, CA, USA) according to the manufacturer's instructions. RT-PCR detection of mRNA expression of survivin and $\beta$-actin (as an internal control) was performed with ProSTAR High Fidelity single-tube RT-PCR system (Stratagene, La Jolla, CA, USA) as described (28). Protein extracts were prepared as described (27). For Western blotting, $\sim 20 \mu \mathrm{g}$ of protein were separated by $10 \%$ sodium dodecyl sulfate polacrylamine gel electrophoresis (SDS-PAGE), transferred onto nitrocellulose membranes (Millipore, Bedford, MA, USA), and visualized by enhanced chemiluminescence (Amersham, Piscataway, NJ, USA) using survivin polyclonal antibody (PAb) c-19 (Santa Cruz Biotechnology, Santa Cruz, CA, USA) or B-actin PAb c-2 (Santa Cruz, as an internal control).

Immunohistochemistry. Immunohistochemistry was executed as described (31). Used primary antibodies included survivin PAb c-19, proliferating cell nuclear antigen (PCNA) monoclonal antibody (MAb) PC10 (Santa Cruz) and factor VIII related antigen (FVIIIRAg) PAb c-20 (Santa Cruz). Percentage of PCNA-positive cells as the proliferative index (PI) and the absolute number of microvessels as the microvessel density (MVD), which were respectively evaluated as described (31).

Cell growth and colony forming assays. For cell growth assay, exponentially growing cells were seeded into 24-well plates $\left(1 \times 10^{4}\right.$ cells per well $)$, cells from triplicate wells were collected every day for consecutive 7 days, cell numbers were determined using a Coulter counter (Coulter Electronics, Miami, FL, USA), and the growth curve of cells was drawn. For colony forming assay, cells were seeded into six-well plates $\left(2 \times 10^{2}\right.$ cells per well), after 12 days of culture, cell colonies were fixed with methanol, stained with Giemsa solution, and counted under a dissection microscope (the colonies containing at least 50 cells were considered viable).

Flow cytometry (FCM) and transmission electron microscopy (TEM) assays. For FCM assay, cells were fixed with $70 \%$ ethanol, incubated with $100 \mu 1$ of RNase I (Sigma-Aldrich, St. Louis, MO, USA) and $100 \mu 1$ of propidium iodide (Sigma-Aldrich), and analyzed by a flow cytometer (Coulter Electronics). The cell cycle phase distribution was calculated and the cells with a subdiploid DNA content were considered apoptotic cells. For TEM assay, cells were pre-fixed with $2.5 \%$ glutaraldehyde, post-fixed with $1 \%$ osmium tetroxide, dehydrated through a graded series of ethyl alcohols, embedded in Epon 618 mixture, cut to $70 \mathrm{~nm}$ thickness serial sections, stained for contrast with uranyl acetate and lead citrate, and observed under JEM-100SX transmission electron microscope (Jeol, Tokyo, Japan). 
Hoechst staining and TdT-mediated dUTP nick end labeling (TUNEL). Cells were stained with Hoechst 33258 solution (Sigma-Aldrich) after the manufacturer's instructions and observed under a fluorescence microscope (Leica, Wetzler, Germany). TUNEL analysis was undertaken with the In Situ Cell Death Detection Kit (Roche Molecular Biochemicals, Mannheim, Germany), sections were observed under a fluorescence microscope (Leica), and percentage of apoptotic cells was regarded as the apoptotic index (AI) as previously described (31).

Glioma xenografts in nude mice. Cells $\left(5 \times 10^{6}\right.$ cells in $100 \mu 1$ of PBS) were implanted by subcutaneous injection into the left flank of each 4-week-old (20-22 g) female athymic BALB/c nude mouse (Center of Laboratory Animal, Fourth Military Medical University, Xi'an, China), to establish the glioma xenografts, each group contained at least five mice. After inoculation, animals were observed every day, tumor size was determined by measuring two diameters perpendicular to each other with a caliper every 5 days. Tumor volume (V) was estimated by using the equation $\mathrm{V}=\mathrm{L}_{\mathrm{x} \mathrm{W}} \mathrm{W}^{2} \mathrm{x} 0.5$, where $\mathrm{L}$ is the mid-axis length and $\mathrm{W}$ is the mid-axis width. Forty-five days after implantation, animals were humanely sacrificed, tumor masses were rapidly removed and weighed, then fixed in $10 \%$ formalin, embedded in paraffin, and subjected to H\&E, TUNEL, or immunohistochemical staining. The protocol of animal experiments was approved by the Committee on the Use of Live Animals in Teaching and Research, Fourth Military Medical University, and institutional guidelines were followed in handling the animals.

Statistical analysis. The outcome variables were expressed as mean \pm standard deviation (SD). Statistical analyses were performed using SPSS 13.0 package (SPSS, Chicago, IL, USA). Differences of variables in multiple groups were first compared using one-way analysis of variance (ANOVA), then the least significant difference (LSD) test was used for comparison of differences between two groups in ANOVA. All statistical tests were two-sided, $\mathrm{P}$ values $<0.05$ were considered statistically significant.

\section{Results}

Specific knockdown of survivin expression in U251 cells by shRNA. According to survivin cDNA sequence, three pairs of specific RNAi fragments targeting survivin were designed and synthesized, which were cloned into pWH1 plasmid vector, and named pWH1-SR1, pWH1-SR2 and pWH1-SR3 vectors respectively. Meanwhile, a non-specific shRNA vector $\mathrm{pWH} 1-\mathrm{NR}$ derived from scrambled specific fragment 2 acting as a control was also constructed. The above vectors were respectively transiently transfected into U251 cells. The results of RT-PCR and Western blotting showed that all the three specific shRNAs markedly inhibited the expressions of mRNA and protein of survivin in U251 cells, whereas the non-specific shRNA did not have this effect (Fig. 1A and B). The pWH1-SR2 vector had the strongest inhibitory effect, and chosen for the permanent transfection analyses. It showed that the survivin expression was further suppressed in the stable transfectant U251-SR transfected
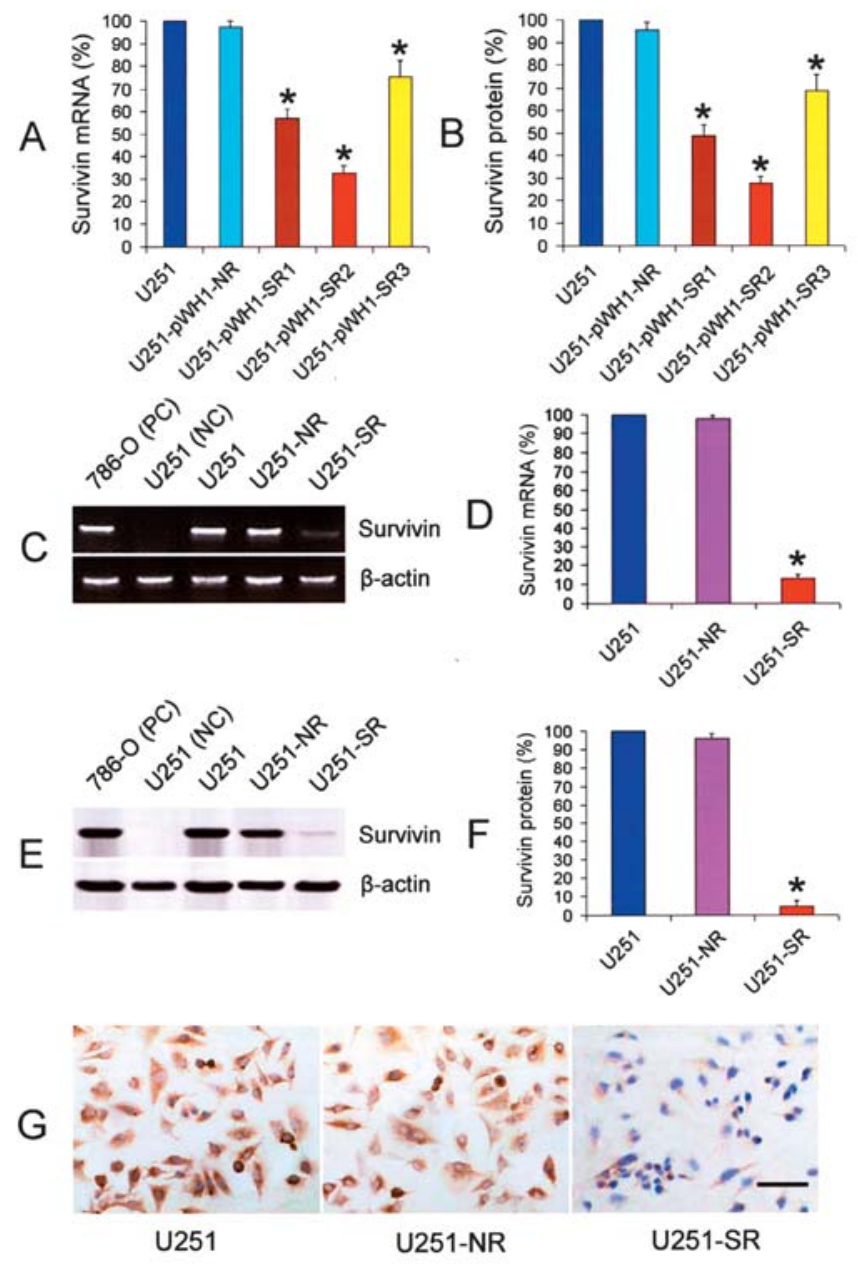

Figure 1. Inhibition of survivin expression by shRNAs in U251 cells. (A) Percentage of survivin mRNA expression in transiently transfected U251 cells. Survivin mRNA was detected with RT-PCR $72 \mathrm{~h}$ after transfection of shRNA vectors; ${ }^{*} \mathrm{P}<0.01$ vs parent U251 cells. (B) Percentage of survivin protein expression in transiently transfected U251 cells. Survivin protein was detected with Western blotting $72 \mathrm{~h}$ after transfection of shRNA vectors; ${ }^{*} \mathrm{P}<0.01$ vs parent $\mathrm{U} 251$ cells. (C) RT-PCR analysis of survivin mRNA expression in stable transfectants. Kidney cancer 786-O cells overexpressing survivin mRNA as a positive control (PC), RNA templates of U251 cells were omitted in RT-PCR as a negative control (NC), and B-actin as an internal control. (D) Percentage of survivin mRNA expression in stable transfectants. ${ }^{*} \mathrm{P}<0.01$ vs parent $\mathrm{U} 251$ cells. (E) Western blot analysis of survivin protein expression in stable transfectants. Kidney cancer 786-O cells overexpressing survivin protein as a positive control (PC), survivin primary antibody was substituted with blocking solution in Western blotting as a negative control (NC), and $\beta$-actin as an internal control. (F) Percentage of survivin protein expression in stable transfectants. ${ }^{*} \mathrm{P}<0.01$ vs parent U251 cells. (G) Immunohistochemistry detection of survivin protein expression in stable transfectants. Original magnification x 100; bar, $50 \mu \mathrm{m}$.

with pWH1-SR2 vector, whereas there was not obvious change in survivin expression in the stable transfectant U251NR transfected with non-specific pWH1-NR vector (Fig. 1C, $\mathrm{D}, \mathrm{E}, \mathrm{F}$ and $\mathrm{G})$. Taken together, the vector expressing shRNA against survivin exerted a powerful and specific knockdown effect with respect to endogenous survivin expression in U251 cells by either transient transfection or stable transfection.

Inhibition of growth of U251 cells in vitro by knockdown of survivin. The results of cell growth assay showed that U251-SR 

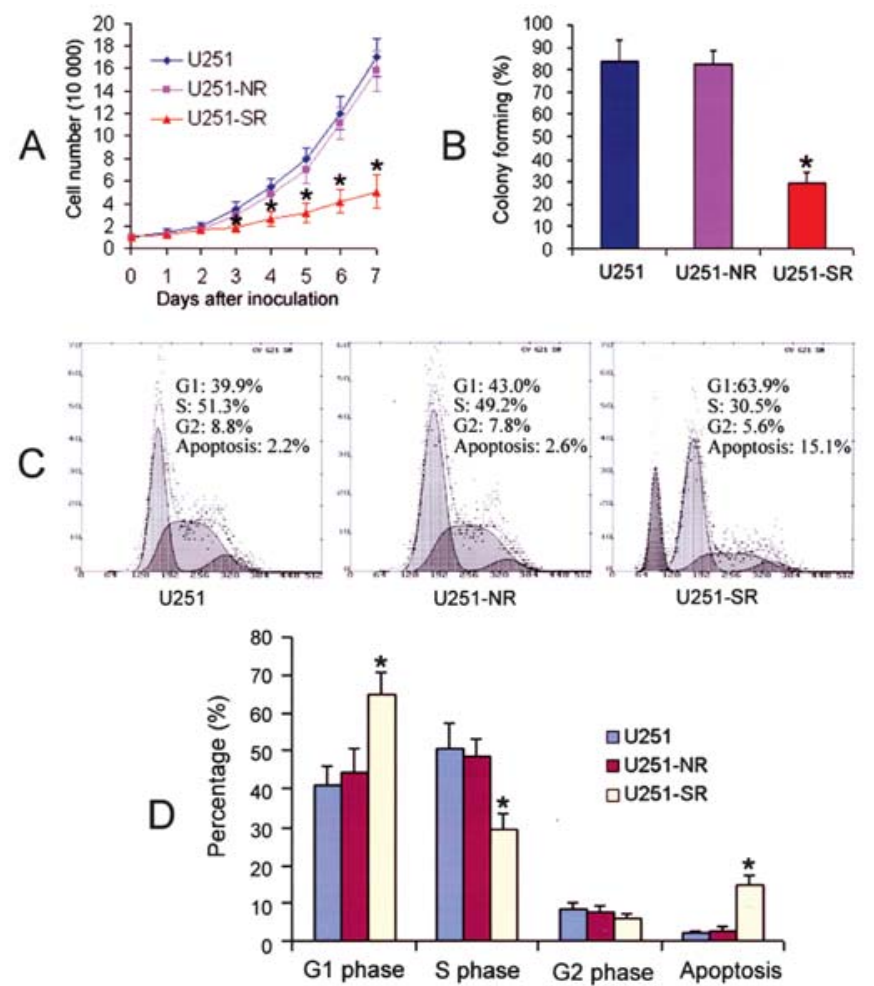

Figure 2. Inhibition of growth and cell cycle progression and induction of apoptosis of U251 cells in vitro by knockdown of survivin. (A) Growth curve of cells. ${ }^{*} \mathrm{P}<0.01$ vs U251 and U251-NR cells. (B) Percentage of colony forming. ${ }^{*} \mathrm{P}<0.01$ vs U251 and U251-NR cells. (C) Representative flow cytometry analysis of cell cycle and cell apoptosis. (D) Percentage of G1, S and G2 phase cells and apoptotic cells. ${ }^{*} \mathrm{P}<0.01$ vs U251 and U251-NR cells.

cells grew significantly slower than parent U251 cells and U251-NR cells, 7 days after plating, the number of cells was reduced by $72.0 \pm 4.0 \%$ in U251-SR cells, as compared with parental U251 cells (Fig. 2A). The results of plate colony forming assay showed that the colony forming percentage in U251-SR cells markedly decreased compared to that in parent U251 cells and U251-NR cells (Fig. 2B). The above results indicated that the growth of $\mathrm{U} 251$ cells in vitro was remarkably inhibited following survivin knockdown.

Induction of apoptosis of U251 cells in vitro by knockdown of survivin. The results of FCM assay showed that apoptotic cells in U251-SR cells significantly increased compared to those in parent U251 cells and U251-NR cells, with an increase of about 6-fold as compared with parent U251 cells (Fig. 2C and D). The apoptotic cells in U251-SR cells presented typical morphologic changes of cell apoptosis, such as, cell shrinkage and round shape, cell membrane bubbled, refractivity of cells increased under phase-contrast microscope (Fig. 3A); chromatin condensed and nuclei were strongly stained in H\&E (Fig. 3B); chromatin collapsed into pieces, nuclei presented bright blue fluorescence in Hoechst staining (Fig. 3C) and bright green fluorescence in TUNEL staining (Fig. 3D); apoptotic cells finally broke into a number of apoptotic bodies (Fig. 3E). The above results indicated that survivin downregulation obviously induced spontaneous apoptosis of U251 cells in vitro.

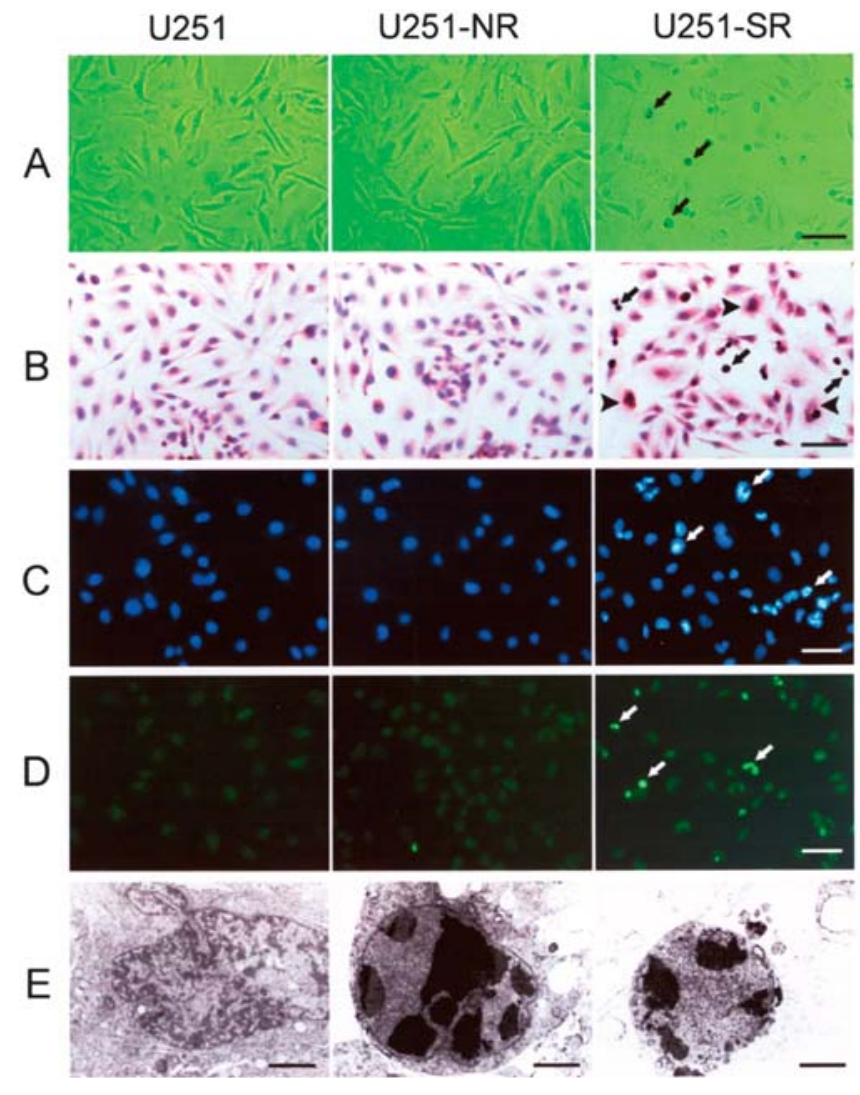

Figure 3. Morphologic changes of U251 cells following survivin knockdown. (A) Phase-contrast microscopy. Black arrows indicate increased apoptotic cells in U251-SR cells; original magnification x100; bar, $50 \mu \mathrm{m}$. (B) H\&E staining. Black arrows indicate increased apoptotic cells, while black arrowheads indicate increased aberrant cells in U251-SR cells; original magnification x 100; bar, $50 \mu \mathrm{m}$. (C) Hoechst staining. White arrows indicate increased apoptotic cells in U251-SR cells; original magnification x200; bar, $35 \mu \mathrm{m}$. (D) TUNEL analysis. White arrows indicate increased apoptotic cells in U251-SR cells; original magnification x200; bar, $35 \mu \mathrm{m}$. (E) Transmission electron microscopy. Left, a normal U251 cell; original magnification x5,000; bar, $2 \mu \mathrm{m}$; middle, an apoptotic U251-SR cell, its chromatin condensed and collapsed into pieces; original magnification, x6,000; bar, $1 \mu \mathrm{m}$; right, an apoptotic body from U251-SR cells; original magnification $\times 10,000$; bar, $0.5 \mu \mathrm{m}$.

Cell cycle arrest and mitotic catastrophe in U251 cells in vitro following knockdown of survivin. The results of cell cycle analysis by FCM showed increased G1 phase and decreased $\mathrm{S}$ phase cells in U251-SR cells than those in parent U251 cells and U251-NR cells. Although there was not a significant difference in G2 phase cells among parent U251, U251-NR and U251-SR cells, there was a mild decreasing trend of G2 phase in U251-SR cells (Fig. 2C and D). The above results suggested that survivin knockdown resulted in marked cell cycle arrest in U251 cells. Furthermore, more of the U251-SR cells $(6.5 \pm 1.8 \%)$ became larger and flatter, and formed multinucleated or giant nucleus cells (Fig. 3B), as compared with parent U251 (1.6 $\pm 1.0 \%)$ and U251-NR cells $(1.9 \pm 1.2 \%)$; the so-called aberrant cells as described above increased about 3-fold in U251-SR cells as compared with parent U251 cells. This result indicated that survivin knockdown led to remarkable mitotic catastrophe in U251 cells.

Inhibition of tumorigenesis in nude mouse glioma xenografts by knockdown of survivin. We then investigated whether 
A
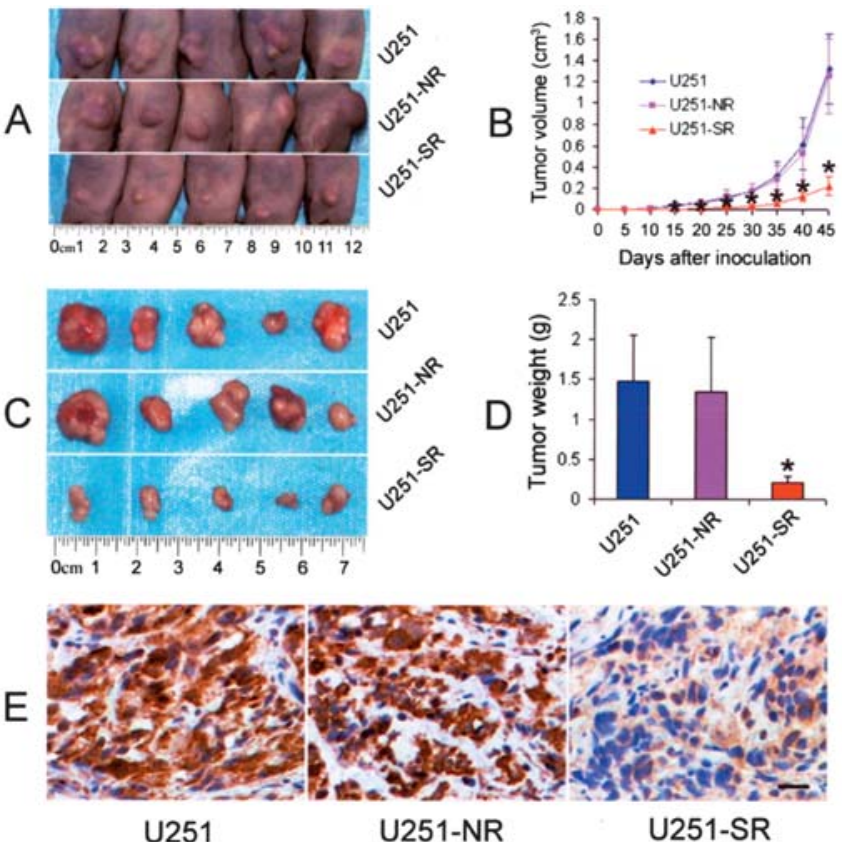

Figure 4. Inhibition of glioma xenograft growth in nude mice in vivo by knockdown of survivin. (A) Tumors in nude mice 45 days after inoculation of cells. (B) Tumor growth curve. ${ }^{*} \mathrm{P}<0.01$ vs $\mathrm{U} 251$ and U251-NR groups. (C) Resected tumor masses 45 days after inoculation of cells. (D) Tumor weight measurement. ${ }^{*} \mathrm{P}<0.01$ vs $\mathrm{U} 251$ and U251-NR groups. (E) Immunohistochemistry detection of survivin protein expression in tumors. Original magnification $\mathrm{x} 400$; bar, $10 \mu \mathrm{m}$.

stable transfectant U251-SR in which survivin expression has been knocked down reduced tumorigenicity in nude mice. For this, parent U251, U251-NR and U251-SR cells were inoculated respectively into nude mice to establish xenograft models of human glioma, the tumor growth status was observed and tumor volume was measured termly. The palpable tumor nodules occurred 10 days after transplantation in all mice inoculated with parent U251 or U251NR cells, and these tumors grew rapidly. Whereas, in mice inoculated with U251-SR cells, the palpable tumor nodules occurred only 3 weeks after transplantation, and they grew slowly. Both tumor volume and tumor weight in U251-SR group were significantly less than those in parent U251 and U251-NR groups (Fig. 4A-D). According to tumor weight, the tumorigenicity was inhibited by $85.6 \pm 8.2 \%$ in U251-SR group as compared with parent U251 group. Furthermore, the immunohistochemistry results showed that survivin protein expression was obviously downregulated in U251-SR group tumors in comparison with that in parent U251 and U251-NR group tumors (Fig. 4E). The above results suggested that shRNA still could efficiently and permanently suppress survivin expression in U251 cells in nude mice in vivo as it did in culture cells in vitro, thus inhibited glioma formation.

Inhibition of cell proliferation and angiogenesis and induction of cell apoptosis and mitotic catastrophe in glioma xenografts by knockdown of survivin. To analyze the mechanisms involved in xenograft growth inhibition mediated by survivin suppression, a series of assays were performed for each group of tumor specimens. It showed the decreased PI and MVD, whereas increased AI and aberrant cells in U251-SR

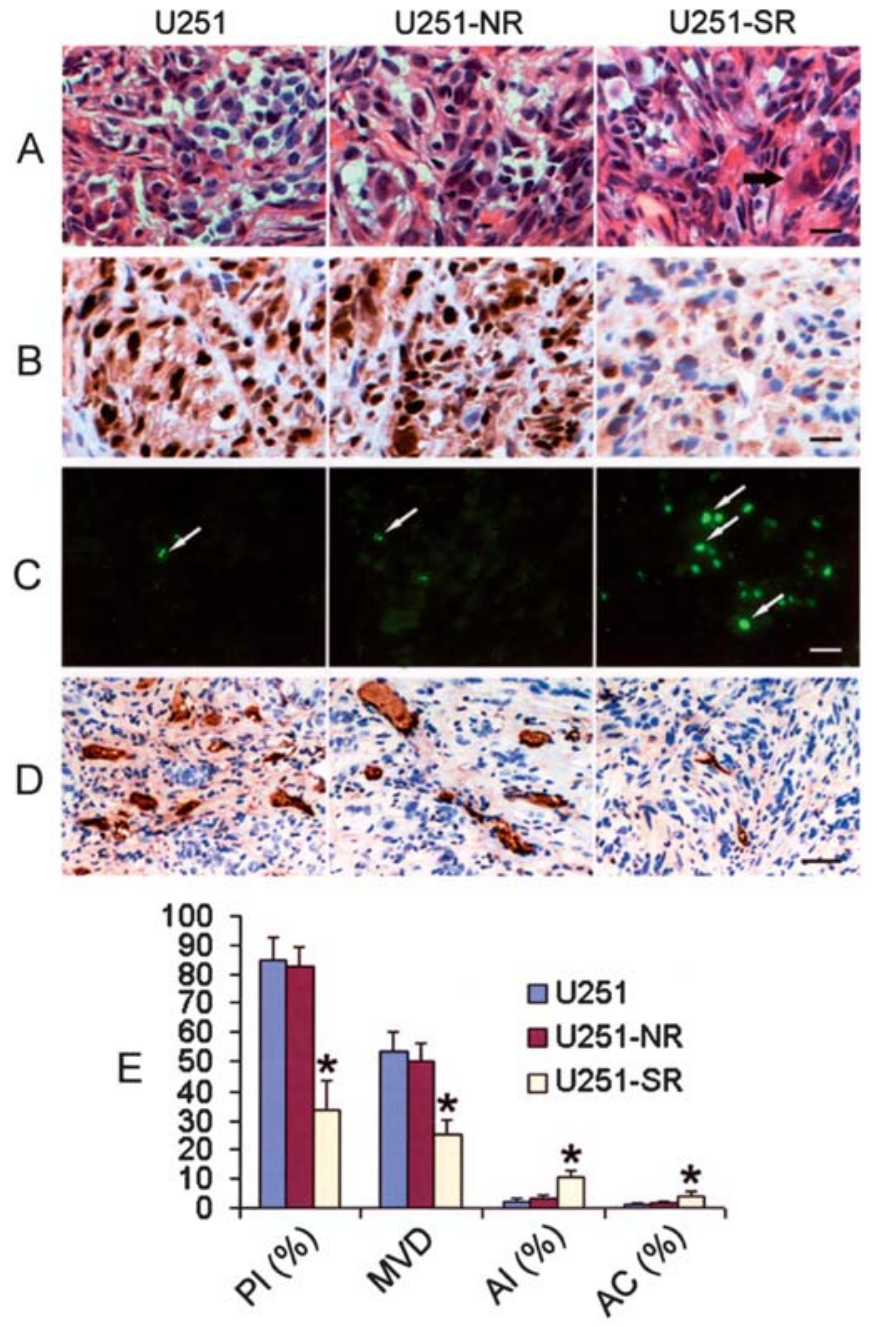

Figure 5. Inhibition of cell proliferation and angiogenesis and induction of cell apoptosis and mitotic catastrophe in glioma xenografts by knockdown of survivin. (A) H\&E staining. Black arrow indicates an aberrant cell; original magnification $\mathrm{x} 400$; bar, $10 \mu \mathrm{m}$. (B) Immunohistochemistry detection of PCNA protein expression. Original magnification $\mathrm{x} 400$; bar, $10 \mu \mathrm{m}$. (C) TUNEL staining. White arrows indicate apoptotic cells; original magnification $\mathrm{x} 400$; bar, $10 \mu \mathrm{m}$. (D) Immunohistochemistry detection of FVIIIRAg protein expression. Original magnification x200; bar, $20 \mu \mathrm{m}$. (E) PI, MVD, AI and percentage of aberrant cells (AC) in tumors. ${ }^{*} \mathrm{P}<0.01$ vs U251 and U251-NR groups.

group tumors, as compared with those in parent U251 and U251-NR group tumors. Both PI and MVD decreased more than one-fold, whereas AI and aberrant cells increased respectively about 4-fold and 2-fold in U251-SR group tumors, in comparison with those in parent U251 group tumors (Fig. 5). The above results indicated that tumorigenesis inhibition following survivin knockdown was attributed to increased cell apoptosis, elicited mitotic catastrophe, compromised cell proliferation and reduced angiogenesis.

\section{Discussion}

RNAi mediated by small interfering RNA (siRNA) has become an excellent tool for exploring gene function in basic research, and may become a novel promising strategy for cancer gene therapy, because of its high efficiency and specificity in blocking target gene expression (32-36). The 
vector-based siRNA expression systems reported so far may be roughly divided into two types, i.e. tandem-type and hairpin-type. In a tandem-type vector, the sense and antisense strands composing the siRNA duplex are independently transcribed by two promoters. While, in a hairpin-type vector, a single promoter drives siRNA expression as a fold-back stem-loop structure that produces effective siRNA after intramolecular processing in cells mediated by Dicer enzyme, a member of the RNase III protein family. The promoters used in driving siRNA transcription mainly include the U6 and $\mathrm{H} 1$ promoters $(32,33,35)$. Although the efficacy of above mentioned vector systems and promoters remain to be further compared, we employed a U6-driven hairpin-type siRNA expression construct in this study, and it showed that the shRNAs efficiently and significantly suppressed the mRNA and protein expressions of survivin in glioma U251 cells.

We showed here that downregulation of survivin expression by shRNA remarkably elicited spontaneous apoptosis, cell cycle arrest, mitotic catastrophe and growth inhibition in U251 cells. These results may be attributed to interfering the important roles of survivin in antiapoptosis, promoting cell proliferation and maintaining mitosis. Up to now, most studies show that survivin is able to bind to caspase-3, caspase-7 and caspase-9, thereby inhibits apoptosis through suppression of caspase activities $(9,10)$. Although few reports show survivin can not bind to caspase-3 (37), and comparison of the X-ray crystallographic structure of survivin with that of the XIAP BIR2-caspase-3 complex fails to suggest how survivin might suppress caspase-3 directly (8). It has been demonstrated that survivin also can bind to the proapoptotic mitochondrial protein Smac/DIABLO, both in vitro and in vivo, this suggests that binding of survivin to Smac/DIABLO may reduce Smac/DIABLO antagonism to other IAPs, such as XIAP, therefore enabling free XIAP to block caspases directly (11). In addition, as a microtubule stability protein and a chromosomal passenger protein, survivin is localized to components of the mitotic apparatus in mitosis process, participates in chromosome segregation and cytokinesis, thus is vitally involved in controlling normal cell division $(12,13)$. Survivin also can initiate cell cycle entry by the competitive interaction with Cdk4/p16 (INK4a) and Cdk2/cyclin E complex activation (14). Recently, Uchida et al (38) showed a tandem-type siRNA targeting survivin induced apoptosis and attenuated growth in HeLa, U251, and MCF-7 cells. Temme et al (39) showed that survivin Thr34Ala mutant elicited a marked decrease in cell proliferation and increase in cell apoptosis in glioma U373 and $\mathrm{H} 4$ cells. Combining our results, it suggests that survivin indeed plays a critical role in antiapoptosis and promoting cell proliferation in gliomas.

The present study showed for the first time that suppression of survivin expression attenuated the angiogenesis in glioma xenografts. This is in line with previous reports showing that survivin may play an important role in promoting angiogenesis. It has been showed that survivin is upregulated in newly formed blood vessel endothelium in vitro and in vivo, but almost undetectable in quiescent endothelial cells. Moreover, a great number of angiogenic agents, such as vascular endothelial growth factor (VEGF), basic fibroblast growth factor (bFGF), angio- poietin-1 (Ang-1) and platelet-derived growth factor (PDGF), can induce survivin overexpression in endothelial cells (1517). Survivin upregulation is one of the important mechanisms for drug 'resistance' in both tumor cells and blood vessel endothelial cells, the suppression of survivin during angiogenesis thus removes its protective effect on endothelial cells, thereby resulting in endothelial apoptosis and destroying the three-dimensional capillary-like vessels in vitro $(16,17)$. The present results strongly indicate that survivin plays a pivotal role in the glioma angiogenesis process, thus, targeting survivin therapeutically will not only directly compromise survival of the glioma cells themselves, but also exert an additional anti-tumor effect by inhibition of the angiogenesis development.

In conculsion, our results show that knockdown of survivin expression by shRNA significantly inhibits the growth of glioma cells in vitro and in vivo, the mechanisms involved include inducing cell apoptosis, eliciting cell cycle arrest, causing mitotic catastrophe, and attenuating angiogenesis. It suggests that survivin has a pleiotropic role in initiation and progression of gliomas, and strongly indicates that survivin may become an attractive target for gene therapy of gliomas. In view of the prominent efficiency and specificity of RNAi mediated by siRNA, such as shRNA, in blocking target gene expression, this may be useful in gene therapy of cancers including gliomas.

\section{Acknowledgments}

This research was supported in part by the National Natural Science Foundation (No. 30672371), Beijing, P.R. China. The authors thank Professor Lei Shang for statistical direction, thank Dr Xiao-Kang Gao for the kidney cancer 786-O cells, and thank Miss Jun-Li Huo for experimental preparation.

\section{References}

1. Brown JM and Attardi LD: The role of apoptosis in cancer development and treatment response. Nat Rev Cancer 5: 231-237, 2005.

2. Hicklin DJ and Ellis LM: Role of the vascular endothelial growth factor pathway in tumor growth and angiogenesis. J Clin Oncol 23: 1011-1027, 2005.

3. Wright CW and Duckett CS: Reawakening the cellular death program in neoplasia through the therapeutic blockade of IAP function. J Clin Invest 115: 2673-2678, 2005.

4. Ambrosini G, Adida C and Altieri DC: A novel anti-apoptosis gene, survivin, expressed in cancer and lymphoma. Nat Med 3: 917-921, 1997.

5. Altieri DC: Validating survivin as a cancer therapeutic target. Nat Rev Cancer 3: 46-54, 2003.

6. Li F and Brattain MG: Role of the survivin gene in pathophysiology. Am J Pathol 169: 1-11, 2006.

7. Duffy MJ, O'Donovan N, Brennan DJ, Gallagher WM and Ryan BM: Survivin: a promising tumor biomarker. Cancer Lett 249: 49-60, 2007

8. Verdecia MA, Huang H, Dutil E, Kaiser DA, Hunter T and Noel JP: Structure of the human anti-apoptotic protein survivin reveals a dimeric arrangement. Nat Struct Biol 7: 602-608, 2000.

9. Shin S, Sung BJ, Cho YS, Kim HJ, Ha NC, Hwang JI, Chung CW, Jung YK and Oh BH: An anti-apoptotic protein human survivin is a direct inhibitor of caspase-3 and -7 . Biochemistry 40 : $1117-1123,2001$.

10. O'Connor DS, Wall NR, Porter AC and Altieri DC: A p34 (cdc2) survival checkpoint in cancer. Cancer Cell 2: 43-54, 2002 . 
11. Sun C, Nettesheim D, Liu Z and Olejniczak ET: Solution structure of human survivin and its binding interface with Smac/Diablo. Biochemistry 44: 11-17, 2005.

12. Vong QP, Cao K, Li HY, Iglesias PA and Zheng Y: Chromosome alignment and segregation regulated by ubiquitination of survivin. Science 310: 1499-1504, 2005.

13. Vader G, Kauw JJ, Medema RH and Lens SM: Survivin mediates targeting of the chromosomal passenger complex to the centromere and midbody. EMBO Rep 7: 85-92, 2006.

14. Suzuki A, Hayashida M, Ito T, Kawano H, Nakano T, Miura M, Akahane K and Shiraki K: Survivin initiates cell cycle entry by the competitive interaction with Cdk4/p16 (INK4a) and Cdk2/cyclin E complex activation. Oncogene 19: 3225-3234, 2000.

15. Mesri M, Morales-Ruiz M, Ackermann EJ, Bennett CF, Pober JS, Sessa WC and Altieri DC: Suppression of vascular endothelial growth factor-mediated endothelial cell protection by survivin targeting. Am J Pathol 158: 1757-1765, 2001.

16. Tran J, Master Z, Yu JL, Rak J, Dumont DJ and Kerbel RS A role for survivin in chemoresistance of endothelial cells mediated by VEGF. Proc Natl Acad Sci USA 99: 4349-4354, 2002.

17. Blanc-Brude OP, Yu J, Simosa H, Conte MS, Sessa WC and Altieri DC: Inhibitor of apoptosis protein survivin regulates vascular injury. Nat Med 8: 987-994, 2002.

18. McKay TR, Bell S, Tenev T, Stoll V, Lopes R, Lemoine NR and McNeish IA: Procaspase 3 expression in ovarian carcinoma cells increases survivin transcription which can be countered with a dominant-negative mutant, survivin T34A; a combination gene therapy strategy. Oncogene 22: 3539-3547, 2003.

19. Kanwar JR, Shen WP, Kanwar RK, Berg RW and Krissansen GW: Effects of survivin antagonists on growth of established tumors and B7-1 immunogene therapy. J Natl Cancer Inst 93: 1541-1552, 2001.

20. Olie RA, Simoes-Wust AP, Baumann B, Leech SH, Fabbro D, Stahel RA and Zangemeister-Wittke U: A novel antisense oligonucleotide targeting survivin expression induces apoptosis and sensitizes lung cancer cells to chemotherapy. Cancer Res 60: 2805-2809, 2000.

21. Leech SH, Olie RA and Zangemeister-Wittke U: Use of antisense oligonucleotides for therapy. Manipulation of apoptosis inhibitors for destruction of lung cancer cells. Methods Mol Med 75: 655-670, 2003.

22. Pennati M, Colella G, Folini M, Citti L, Daidone MG and Zaffaroni N: Ribozyme-mediated attenuation of survivin expression sensitizes human melanoma cells to cisplatin-induced apoptosis. J Clin Invest 109: 285-286, 2002.

23. Pennati M, Binda M, Colella G, Zoppe' M, Folini M, Vignati S, Valentini A, Citti L, De Cesare M, Pratesi G, Giacca M, Daidone MG and Zaffaroni N: Ribozyme-mediated inhibition of survivin expression increases spontaneous and drug-induced apoptosis and decreases the tumorigenic potential of human prostate cancer cells. Oncogene 23: 386-394, 2004.

24. Ning S, Fuessel S, Kotzsch M, Kraemer K, Kappler M, Schmidt U, Taubert H, Wirth MP and Meye A: siRNA-mediated down-regulation of survivin inhibits bladder cancer cell growth. Int J Oncol 25: 1065-1071, 2004.
25. Ai Z, Yin L, Zhou X, Zhu Y, Zhu D, Yu Y and Feng Y: Inhibition of survivin reduces cell proliferation and induces apoptosis in human endometrial cancer. Cancer 107: 746-756, 2006

26. Phillips HS, Kharbanda S, Chen R, Forrest WF, Soriano RH, Wu TD, Misra A, Nigro JM, Colman H, Soroceanu L, Williams PM, Modrusan Z, Feuerstein BG and Aldape K: Molecular subclasses of high-grade glioma predict prognosis, delineate a pattern of disease progression, and resemble stages in neurogenesis. Cancer Cell 9: 157-173, 2006.

27. Chakravarti A, Noll E, Black PM, Finkelstein DF, Finkelstein DM, Dyson NJ and Loeffler JS: Quantitatively determined survivin expression levels are of prognostic value in human gliomas. J Clin Oncol 20: 1063-1068, 2002.

28. Kajiwara Y, Yamasaki F, Hama S, Yahara K, Yoshioka H, Sugiyama K, Arita K and Kurisu K: Expression of survivin in astrocytic tumors: correlation with malignant grade and prognosis. Cancer 97: 1077-1083, 2003

29. Yamada Y, Kuroiwa T, Nakagawa T, Kajimoto Y, Dohi T, Azuma H, Tsuji M, Kami K and Miyatake S: Transcriptional expression of survivin and its splice variants in brain tumors in humans. J Neurosurg 99: 738-745, 2003.

30. Jiao BH, Yao ZG, Geng SM and Zuo SH: Expression of survivin, a novel apoptosis inhibitor and cell cycle regulatory protein, in human gliomas. Chin Med J (Engl) 117: 612-614, 2004

31. Zhen HN, Zhang X, Hu PZ, Yang TT, Fei Z, Zhang JN, Fu LA, He XS, Ma FC and Wang XL: Survivin expression and its relation with proliferation, apoptosis, and angiogenesis in brain gliomas. Cancer 104: 2775-2783, 2005.

32. Mittal V: Improving the efficiency of RNA interference in mammals. Nat Rev Genet 5: 355-365, 2004

33. Stevenson M: Therapeutic potential of RNA interference. N Engl J Med 351: 1772-1777, 2004.

34. Shankar P, Manjunath $\mathrm{N}$ and Lieberman J: The prospect of silencing disease using RNA interference. JAMA 293: 1367-1373, 2005.

35. Moffat J and Sabatini DM: Building mammalian signalling pathways with RNAi screens. Nat Rev Mol Cell Biol 7: 177-187, 2006.

36. Kim DH and Rossi JJ: Strategies for silencing human disease using RNA interference. Nat Rev Genet 8: 173-184, 2007.

37. Banks DP, Plescia J, Altieri DC, Chen J, Rosenberg SH, Zhang H and Ng SC: Survivin does not inhibit caspase-3 activity. Blood 96: 4002-4003, 2000

38. Uchida H, Tanaka T, Sasaki K, Kato K, Dehari H, Ito Y, Kobune M, Miyagishi M, Taira K, Tahara $\mathrm{H}$ and Hamada $\mathrm{H}$ : Adenovirus-mediated transfer of siRNA against survivin induced apoptosis and attenuated tumor cell growth in vitro and in vivo. Mol Ther 10: 162-171, 2004.

39. Temme A, Herzig E, Weigle B, Morgenroth A, Schmitz M, Kiessling A, Rieger MA, Schackert HK and Rieber EP. Inhibition of malignant glioma cell growth by a survivin mutant retrovirus. Hum Gene Ther 16: 209-222, 2005. 